Annette Cina, Guy Bodenmann, Kurt Hahlweg, Thomas

Dirscherl und Matthew R. Sanders

\title{
Triple P (Positive Parenting Program): Theoretischer und empirischer Hintergrund und erste Erfahrungen im deutschsprachigen Raum
}

\author{
Triple P (Positive Parenting Program): Theoretical and empirical \\ background and first experiences in the German speaking areas
}

\begin{abstract}
Zusammenfassung
Dieser Artikel stellt die theoretischen, empirischen und klinischen Grundlagen eines mehrstufigen erziehungs- und familienunterstützenden Präventionsprogramms (Triple P: Positive Parenting Program) dar, welches die Förderung elterlicher Erziehungskompetenzen und die Reduktion von Verhaltensproblemen und emotionalen Störungen bei Kindern und Jugendlichen zum Ziel hat. Der Beitrag stellt die einzelnen Interventionsmethoden, Trainingselemente, Formen von Triple $\mathrm{P}$ und dessen Neuerungen sowie insbesondere die Verbreitung und Implementierung des Elterngruppenprogramms in Deutschland und der Schweiz dar. Die empirischen Belege zur Wirksamkeit des Programms aus Australien sowie aus dem deutschsprachigen Raum werden resümiert und Schlussfolgerungen für die Untersuchung der weiteren Verbreitung des Programms dargestellt.
\end{abstract}

\begin{abstract}
Schlagworte: Triple P, Prävention, Erziehung, kindliches Problemverhalten, Verhaltenstherapie.
\end{abstract}

\section{Abstract}

This article reviews the theoretical and empirical background of Triple P (Positive Parenting Program). The aims of this prevention program as well as the different techniques that are used in order to help parents to improve their educational skills are presented. One main focus of this article is to draw the development of Triple P, its features and specifically its implementation in Europe. It is shown, how Triple P is disseminated in Germany and Switzerland and how parents rate their satisfaction and acceptance of Triple $\mathrm{P}$ in these two countries.

Key words: Triple $\mathrm{P}$, prevention, education, child problem behavior, behavioral therapy

Für das Wohlbefinden von Kindern ist die Qualität des Familienlebens (insbesondere die Eltern-Kind-Beziehung) von zentraler Bedeutung. Viele Probleme bezüglich der psychischen Gesundheit wie auch soziale und wirtschaftliche Probleme stehen in enger Verbindung mit Störungen in der Familie (Chamberlain/Patterson 1995; Patterson 1982; Sanders/Duncan 1995). 
Epidemiologische Studien zeigen, dass familiäre Risikofaktoren wie mangelhafte Erziehungskompetenzen der Eltern, häufige Familienkonflikte oder Scheidung sowie das psychische Befinden der Eltern die Entwicklung des Kindes in starkem Ausmaß beeinflussen (vgl. z.B. Cummings/Davies 1994; Dryfoos 1990; Robins/Price 1991). Besonders das Fehlen einer positiven Beziehung zu den Eltern, inadäquate Erziehungskompetenzen der Eltern, Partnerschaftsstörungen und elterliche Psychopathologien (besonders mütterliche Depression) erhöhen das Risiko für Verhaltensprobleme oder emotionale Störungen (vgl. z.B. Coie 1996; Loeber/Far-rington 1998). Entsprechend wichtig sind präventive Interventionen, die Familien stärken und damit Kindern eine gesunde Entwicklung ermöglichen. Das Programm Triple P (Positive Parenting Program) stellt ein Programm dar, welches auf die Förderung von elterlichen Erziehungskompetenzen fokussiert ist und aufgrund seines mehrstufigen Aufbaus eine gute Möglichkeit darstellt, Interventionen spezifisch auf die individuellen Bedürfnisse der Eltern anzubieten.

\section{Das Triple P-System}

Triple P (Positive Parenting Program) ist ein mehrstufiges, integriertes erziehungsund familienunterstützendes Präventions- und Interventionsprogramm, das von Prof. Matthew R. Sanders und Kollegen an der Universität Queensland in Brisbane, Australien, entwickelt wurde. Das Programm zielt auf die Prävention von, aber auch auf die Intervention bei Verhaltens- und Entwicklungsproblemen sowie emotionalen Problemen ab, indem das Wissen, die Kompetenzen und das Selbstvertrauen von Eltern gestärkt werden. Das Programm richtet sich an Eltern von Kindern von 2 bis 12 Jahren (Triple P Preteen) und 11 bis 16 Jahren (Triple P Teen). Dabei werden fünf verschiedene Entwicklungsstufen der Kindheit bis zur Adoleszenz berücksichtigt. Der Mehr-Ebenen-Charakter des Programms ermöglicht eine Vielzahl von Kombinationen hinsichtlich Interventionsebenen und Interventionsformen (Gruppe, individuell, selbständig) (siehe Tabelle 1). Je nach lokalen Prioritäten, Mitarbeitern und Budgetvorgaben können diese flexibel als universelle, indizierte oder selektive Prävention eingesetzt werden. 
Tabelle 1: Die Triple P-Interventionsebenen

\begin{tabular}{|c|c|c|c|c|c|}
\hline \multicolumn{2}{|c|}{ Interventions-ebene } & \multirow{2}{*}{\begin{tabular}{l|}
\multicolumn{1}{c|}{ Zielgruppe } \\
Alle Eltern, die an \\
Informationen zur \\
Förderung der Ent- \\
wicklung ihres Kindes \\
interessiert sind.
\end{tabular}} & \multirow{2}{*}{$\begin{array}{l}\text { Interventions-methoden } \\
\text { Kurze schriftliche oder } \\
\text { mündliche Information, } \\
\text { Selbsthilfematerialien, } \\
\text { Gruppenpräsentationen, } \\
\text { Einsatz von Medien. }\end{array}$} & \multirow[b]{2}{*}{\begin{tabular}{l}
\multicolumn{1}{c|}{$\begin{array}{l}\text { Materialien } \\
\text { (Deutsch) }\end{array}$} \\
Tip Sheets („Kleine \\
Helfer“) zur Positiven \\
Erziehung \\
Broschüre „Positive \\
Erziehung“ \\
Video „Überlebenshilfe \\
für Eltern“ \\
Triple P-Eltern- \\
arbeitsbuch (Selbsthilfe- \\
buch) \\
\end{tabular}} & \multirow{2}{*}{\begin{tabular}{l}
\multicolumn{1}{c}{$\begin{array}{c}\text { Mögliche Ziel- } \\
\text { verhaltensweisen }\end{array}$} \\
Alltägliche Verhaltens- \\
probleme
\end{tabular}} \\
\hline 1. & $\begin{array}{l}\text { Universelle } \\
\text { Informationen } \\
\text { über Erziehung }\end{array}$ & & & & \\
\hline 2. & $\begin{array}{l}\text { Kurzberatung für } \\
\text { spezifische } \\
\text { Erziehungsprob- } \\
\text { leme }\end{array}$ & $\begin{array}{l}\text { Eltern mit spezifischen } \\
\text { Sorgen um das } \\
\text { Verhalten oder die } \\
\text { Entwicklung } \\
\text { ihres Kindes. }\end{array}$ & $\begin{array}{l}\text { Kurzberatungen mit } \\
\text { konkreten Hinweisen } \\
\text { zum Umgang mit kon- } \\
\text { kreten Verhaltensprob- } \\
\text { lemen. } \\
\text { Kann Face-to-Face } \\
\text { Kontakt, Telefonkontakt } \\
\text { oder Gruppenberatung } \\
\text { sein. }\end{array}$ & $\begin{array}{l}\text { Materialien Ebene } 1 \\
\text { Triple P- } \\
\text { Elternarbeitsbuch } \\
\text { (Selbsthilfebuch) } \\
\text { Poster zu kindlicher } \\
\text { Entwicklung } \\
\text { Beratung-Flip-Chart }\end{array}$ & $\begin{array}{l}\text { Einzelne leichte bis } \\
\text { mittele Problemverhal- } \\
\text { tensweisen ( } \mathrm{z} . \mathrm{B} . \\
\text { Probleme beim Zu- } \\
\text { bettgehen, Probleme } \\
\text { mit Wutanfällen, } \\
\text { Probleme mit Essens- } \\
\text { zeiten, Toilettentrai- } \\
\text { ning) }\end{array}$ \\
\hline 3. & $\begin{array}{l}\text { Kurzberatung für } \\
\text { spezifische } \\
\text { Erziehungsprob- } \\
\text { leme und aktives } \\
\text { Training }\end{array}$ & $\begin{array}{l}\text { Eltern mit spezifischen } \\
\text { Sorgen um das } \\
\text { Verhalten oder die } \\
\text { Entwicklung } \\
\text { ihres Kindes und } \\
\text { Defiziten in Erzie- } \\
\text { hungsfertigkeiten. }\end{array}$ & $\begin{array}{l}\text { Kurzes Programm } \\
\text { ( } 1 \text { bis } 4 \text { Sitzungen) } \\
\text { enthält Ratschläge, } \\
\text { Rollenspiele und Selbst- } \\
\text { einschätzungen, in } \\
\text { denen Eltern lernen } \\
\text { sollen, mit } \\
\text { Verhaltensproblemen } \\
\text { umzugehen. }\end{array}$ & $\begin{array}{l}\text { Materialien Ebene } 1 \\
\text { und } 2\end{array}$ & $\begin{array}{l}\text { Wie bei Ebene } 2 \\
\text { Außerdem andauernde } \\
\text { Essprobleme, Angst- } \\
\text { management o. Ä. }\end{array}$ \\
\hline 4. & $\begin{array}{l}\text { Intensives } \\
\text { Elterntraining }\end{array}$ & $\begin{array}{l}\text { Eltern von Kindern mit } \\
\text { schwerwiegenderen } \\
\text { Verhaltensproblemen. } \\
\text { Eltern mit Erziehungs- } \\
\text { defiziten. } \\
\text { Eltern, die ein intensi- } \\
\text { ves Training positiver } \\
\text { Erziehungsfertigkeiten } \\
\text { möchten. }\end{array}$ & $\begin{array}{l}\text { Intensives Programm, } \\
\text { fokussiert auf Eltern- } \\
\text { Kind-Interaktionen. } \\
\text { Anwendung, Erwei- } \\
\text { terung und Generalisie- } \\
\text { rung von Erziehungsfer- } \\
\text { tigkeiten auf ein breites } \\
\text { Feld von kindlichen } \\
\text { Verhaltensweisen. }\end{array}$ & $\begin{array}{l}\text { Materialien Ebene } 1 \\
\text { bis } 3 \\
\text { Triple P- } \\
\text { Elternarbeitsbuch } \\
\text { (Selbsthilfebuch) } \\
\text { Triple P- } \\
\text { Gruppenarbeitsbuch für } \\
\text { Eltern } \\
\text { Familienarbeitsbuch } \\
\end{array}$ & $\begin{array}{l}\text { Generelle Erziehungs- } \\
\text { sorgen } \\
\text { Aggressives Verhalten } \\
\text { Oppositionelles Prob- } \\
\text { lemverhalten } \\
\text { Verhaltensprobleme } \\
\text { Lernschwierigkeiten }\end{array}$ \\
\hline 5. & $\begin{array}{l}\text { Erweiterte Inter- } \\
\text { ventionen auf } \\
\text { Familienebene }\end{array}$ & $\begin{array}{l}\text { Eltern von Kindern mit } \\
\text { deutlichen Verhal- } \\
\text { tensproblemen oder } \\
\text { Kinder aus Multi- } \\
\text { Problem-Familien. }\end{array}$ & $\begin{array}{l}\text { Intensives Programm mit } \\
\text { zusätzlichen Modulen } \\
\text { wie Hausbesuchen zur } \\
\text { Steigerung der elterli- } \\
\text { chen Erziehungskompe- } \\
\text { tenzen, der Stimmungs- } \\
\text { und Stressmanagement- } \\
\text { strategien, sowie der } \\
\text { Partnerunterstützung. }\end{array}$ & $\begin{array}{l}\text { Materialien Ebene } 1 \\
\text { bis } 4 \\
\text { Arbeitsbuch zu Ebene } 5\end{array}$ & $\begin{array}{l}\text { Andauernde Verhal- } \\
\text { tensstörungen } \\
\text { Zusätzliche Probleme } \\
\text { der Eltern: Z.B. Bezie- } \\
\text { hungskonflikte, De- } \\
\text { pression, Kindesmiss- } \\
\text { handlung }\end{array}$ \\
\hline
\end{tabular}




\section{Theoretischer Hintergrund}

Triple P stellt eine verhaltenstherapeutische Familienintervention dar, welche vornehmlich auf lerntheoretischen Prinzipien basiert. Der Programminhalt greift insbesondere auf Modelle der sozialen Lerntheorie zurück, welche die reziproke und wechselseitige Natur der Eltern-Kind-Interaktion hervorheben (z.B. Patterson 1982). Eltern lernen im Programm Alternativen zu dysfunktionalen Erziehungspraktiken kennen und anwenden. Weiter rekurriert Triple $\mathrm{P}$ auf Modelle der sozialkognitiven Lerntheorie (Bandura 1977, 1995; Dodge 1986; Crick/Dodge 1994), indem die Rolle der elterlichen Kognitionen wie Attributionen, Erwartungen und Überzeugungen bearbeitet werden. Diese Faktoren sind bedeutsam im Zusammenhang mit der elterlichen Selbstwirksamkeit, dem Treffen von Entscheidungen und den Reaktionen der Eltern auf das kindliche Verhalten. Durch die Intervention von Triple $\mathrm{P}$ wird versucht, die Attributionen der Eltern dahingehend zu modifizieren, dass sie günstigere soziale Erklärungen für das Verhalten ihres Kindes finden. Ferner werden bei Triple P Erkenntnisse aus der Entwicklungspsychopathologie, die spezifische Risiko- und Schutzfaktoren für Verhaltensprobleme bei Kindern und Jugendlichen aufzeigen, berücksichtigt (z.B. Emery 1982; Erel/Burman 1995; Grych/Fincham 1990; Hart/Risley 1995; Rutter 1985). Gleichzeitig integriert das Programm Erkenntnisse aus der Entwicklungspsychologie, die die Bedeutung der frühen Eltern-Kind-Interaktion für die soziale und intellektuelle Entwicklung des Kindes aufzeigen (z.B. Hart/Risley 1995; White 1990), Erkenntnisse aus der Erziehungsstilforschung (Grych/Fincham 1990; Rutter 1985) und Partnerschaftsforschung im Zusammenhang mit dem Wohlbefinden von Kindern (Dadds/Schwartz/ Sanders 1987; Jacobson/Margolin 1979).

\section{Interventionsmethoden}

Im Triple P-Programm werden Methoden aus der kognitiven Verhaltenstherapie (Gefühlskontrolle, Veränderung dysfunktionaler Kognitionen und Attributionen sowie spezifische Bewältigungsstrategien für Risikosituationen), Techniken der Selbstkontrolle (z.B. Selbstbeobachtung, Kontingenzkontrolle), Techniken der Konsequenzkontrolle (z.B. operante Verstärkung und Löschung, Kontingenzmanagement, time-out) sowie des Modelllernens eingesetzt. In der Umsetzung greift das Programm zurück auf Forschungsarbeiten der Kinder- und Familienverhaltenstherapie und auf verhaltensanalytische Modelle, welche wirksame Verhaltensänderungsstrategien entwickelt haben. Bedeutsam sind Forschungsergebnisse, die den Fokus auf die Veränderung der auslösenden Bedingungen von Verhaltensweisen legen, indem für eine positive und sichere Lernumgebung der Kinder gesorgt wird (Risley/Clarke/Cataldo 1976; Sanders 1992, 1996). 


\section{Menschenbild bei Triple P}

Triple P geht von einem ganzheitlich-systemischen Menschenbild aus, in dem kognitive, emotionale, motorische und physiologische Faktoren wie auch die Interaktionen einer Person mit ihrer physikalischen und sozialen Umwelt integriert sind. Triple P betont die Bedeutung von Kommunikations- und Interaktionsprozessen in der Familie. Dabei wird der wechselseitigen Beeinflussung von Kind- und Elternverhalten Rechnung getragen. Bedürfnisse von Kindern und ihre Entwicklung zur Selbständigkeit und Förderung der Selbstkontrolle spielen eine wichtige Rolle bei Triple P. Kinder werden je nach Entwicklungsstand und Fähigkeiten in zunehmendem $\mathrm{Ma} ß$ an der aktiven Lebensgestaltung und den Entscheidungen in der Familie beteiligt. Triple P vermittelt Anregungen und Fertigkeiten für die Erziehung, die gemeinsam mit den Eltern verhaltensorientiert umgesetzt und in den Familienalltag integriert werden.

\section{Ziele von Triple P}

Triple P fokussiert auf die Erhöhung der familiären protektiven Faktoren und die Reduzierung von Risikofaktoren, welche mit schweren Verhaltens- und emotionalen Problemen bei Kindern und Jugendlichen verbunden sind. Ziel des Programms ist die Prävention bzw. Reduktion von Störungen bei Kindern, wobei die Intervention bei den Eltern ansetzt (Steigerung des Erziehungsverhaltens mit Hilfe von positiven Erziehungsstrategien). Die Steigerung des Wissens, der Erziehungsfertigkeiten, des Selbstvertrauens in die eigenen Kompetenzen sowie die Förderung einer liebevollen, sicheren, gewaltfreien und konfliktarmen Umgebung für Kinder stellen die Ziele auf Seiten der Eltern dar. Bei den Kindern zielt Triple P auf die Förderung der sozialen, emotionalen, sprachlichen, intellektuellen und Verhaltenskompetenzen der Kinder. Dabei wird berücksichtigt, dass sich Eltern unterscheiden hinsichtlich des Ausmaßes an Intervention und Unterstützung, welche sie benötigen, um selbständig ein Problem bewältigen zu können. Ziel von Triple P ist daher, den Eltern nur soviel Unterstützung zu geben, wie diese auch wirklich benötigen.

Der Fähigkeit der Eltern zur Selbstregulation wird bei Triple P besondere Beachtung geschenkt. Die Förderung von Unabhängigkeit, Selbstmanagement, Selbstattribution und elterlicher Selbstwirksamkeit stehen im Vordergrund. Ziel ist, den Eltern intensiv in kurzer Zeit Kompetenzen zu vermitteln, die ihnen ermöglichen, selbständig Probleme zu lösen (Unabhängigkeit), ihre Erziehungsziele in Abhängigkeit ihrer Fertigkeiten selber festzulegen, zu überprüfen und zu adaptieren (Selbstmanagement) und Veränderungen oder Verbesserungen auf eigene Bemühungen respektive solche des Kindes zurückzuführen anstatt auf Zufall, Reifung oder äußere Ereignisse (Selbstattribution). Ferner sollen die Eltern zur Überzeugung gelangen, dass sie ein Erziehungsproblem bewältigen und lösen können (elterliche Selbstwirksamkeit). Triple P zielt damit sowohl auf behaviorale 
Veränderungen als auch auf die Beeinflussung von kognitiven Prozessen ab und damit verbunden auf die Selbstreflexion der Eltern.

Zielgruppen von Triple P

Das Programm richtet sich an Eltern mit Kindern von Geburt bis 16 Jahren und vermittelt Erziehungsstrategien, -wissen und Selbstvertrauen bezüglich der Erziehung. Das Unterstützungs- und Beratungsangebot von Triple P kann von einer Vielzahl von professionellen Helfern genutzt werden, wie z.B. von Krankenschwestern, Hausärzten, Kinderärzten, Lehrern, Sozialarbeitern, Psychologen, Mütter-Väter-Beratern und Psychiatern.

\title{
Kernprinzipien und Erziehungsstrategien von Triple P
}

Fünf Kernprinzipien der positiven Erziehung bilden die Grundlage des Programms (siehe Tabelle 2). Diese Prinzipien entsprechen spezifischen Schutzfaktoren, die erwiesenermaßen einen positiven Effekt auf die Entwicklung und das psychische Gleichgewicht von Kindern haben.

Tabelle 2: $\quad$ Kernprinzipien einer positiven Erziehung

\begin{abstract}
Für eine sichere und interessante Umwelt sorgen
Kinder jeden Alters brauchen für eine gesunde Entwicklung Beaufsichtigung und ein sicheres Umfeld, welches innen Möglichkeiten zum Erkunden, Experimentieren und Spielen bietet. Dieses Prinzip ist, unter Berücksichtigung des jeweiligen Entwicklungskontextes, auch für ältere Kinder und Jugendliche von Bedeutung.

Eine positive und anregende Lernatmosphäre schaffen

Eltern werden in ihrer Rolle als wichtige Lehrperson ihres Kindes ausgebildet. Das Programm zielt v.a. auf die Förderung der Problemlösefähigkeit der Kinder beispielsweise durch beiläufiges Lernen in verschiedenen Alltagssituationen (z.B. Bitten um Hilfe, Information, Ratschlag, Aufmerksamkeit) ab.
\end{abstract}

\section{Sich konsequent verhalten}

Die Eltern lernen die Grundlagen konsequenten Verhaltens kennen. Dies umfasst, Grundregeln für spezifische Situationen festzulegen, Regeln mit den Kindern zu diskutieren; klare, ruhige, dem Alter angepasste Anweisungen zu geben oder, wenn nötig, absichtliches Ignorieren, logische Konsequenzen, den Stillen Stuhl oder die Auszeit einzusetzen. Diese Strategien stellen wirksame positive Alternativen zu Zwangsprozessen oder wirkungslosen Disziplinierungsverfahren (wie z.B. Anschreien, Drohen oder Körperstrafe) dar.

Realistische Erwartungen haben

Die Eltern erhalten die Möglichkeit, ihre Erwartungen, Annahmen und Meinungen, mit denen sie das kindliche Verhalten erklären, kritisch zu hinterfragen und sich Ziele auszuwählen, die einerseits der kindlichen Entwicklung angemessen und andererseits realistisch für die Eltern sind.

Die eigenen Bedürfnisse beachten

Elternsein wird von verschiedenen Faktoren, die Auswirkungen auf die elterliche Selbstachtung und das Wohlbefinden haben, beeinflusst. Sämtliche Triple P-Ebenen ermuntern Eltern dazu, dem eigenen Wohlbefinden und den eigenen Bedürfnissen Sorge zu tragen und vermitteln ihnen die dafür erforderlichen Erziehungsstrategien. 
Im Programm werden neben Fertigkeiten der Verhaltensbeobachtung, Möglichkeit zur Prävention von Problemen in Risikosituationen, Umgang mit Stimmungsschwankungen, Unterstützung des Partners und Kommunikationsfähigkeiten insbesondere 17 Erziehungsstrategien vermittelt, die Eltern helfen, präventiv problematischem Verhalten des Kindes vorzubeugen sowie adäquat auf das Problemverhalten des Kindes zu reagieren (siehe Tabelle 3).

Tabelle 3: Die Triple P-Erziehungsfertigkeiten

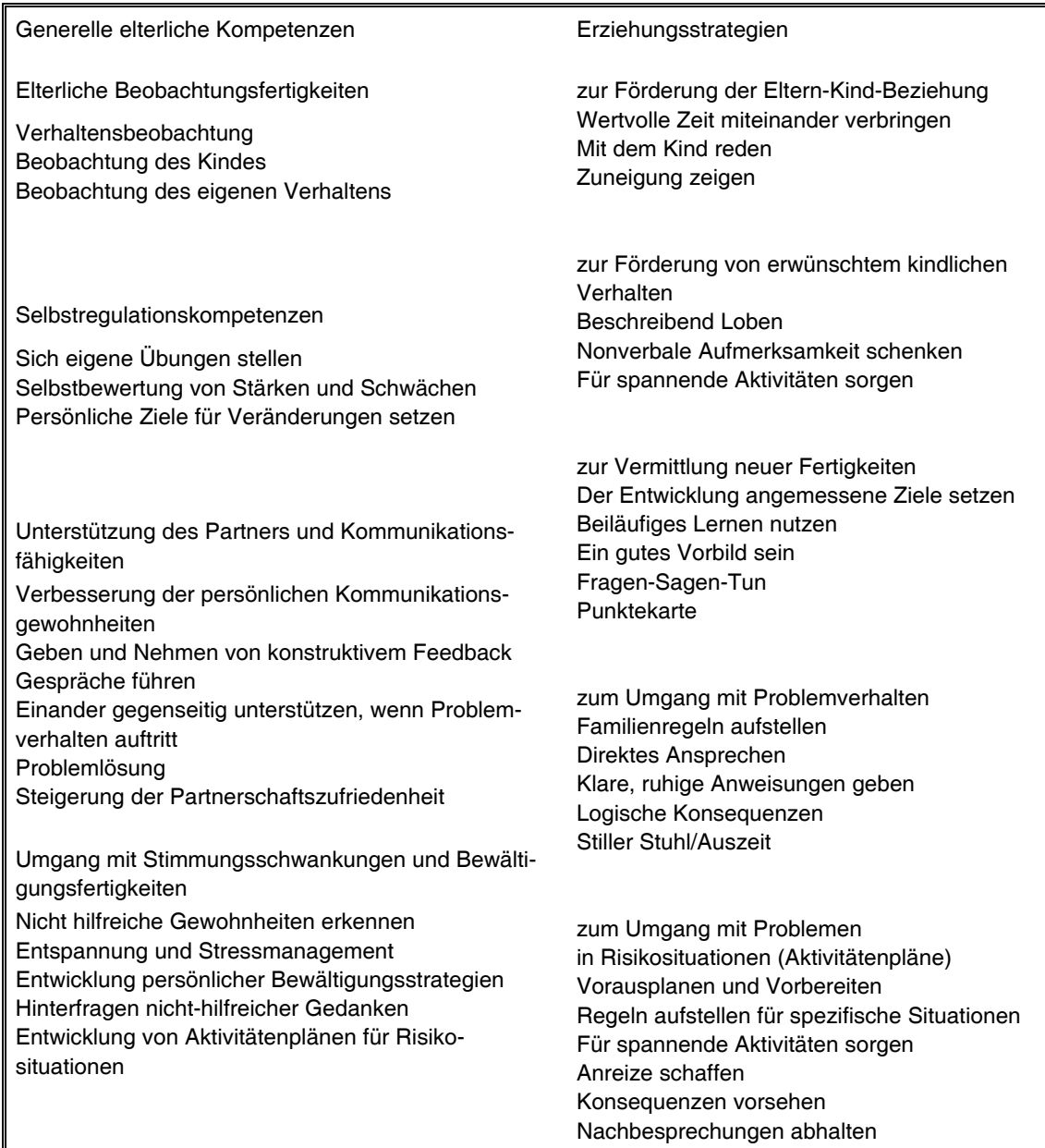




\section{Wirksamkeit von Triple P}

Erste Studien zur Wirksamkeit von Einzelteilen von Triple P als verhaltenstherapeutische Familienintervention (Behavioral Family Intervention, BFI) begannen bereits Ende der 1970er Jahre und wurden in den frühen 1980er Jahren veröffentlicht (z.B. Sanders/Glynn 1981). Seither wurde Triple P kontinuierlich erweitert und in Quer- und Längsschnittstudien (Kontrollgruppendesigns mit Zufallzuordnung) evaluiert (siehe für einen Überblick Sanders/Dadds 1993). Mehrere Studien belegen inzwischen die Effizienz sowie Effektivität von Triple P (z.B. Sanders 1996, 1998; Sanders/Christensen 1985; Sanders/Dadds 1982; Sanders/Glynn 1981; Taylor/Biglan 1998). Zusammenfassend kann gesagt werden, dass sich Triple $P$ als verhaltenstherapeutische Familienintervention zur wirkungsvollen Behandlung von externalisierenden Störungen, insbesondere oppositionellem Trotzverhalten (Forehand/Long 1988; Webster-Stratton 1994), Aufmerksamkeitsdefizit- und Hyperaktivitätsstörung ADHS (Barkley/Guevremont/Anastopoulos/Fletcher 1992), Essproblemen (Turner/Sanders/Wall 1994), Schmerzsyndromen (Sanders/Shepard/Cleg-horn/Woolford 1994), Angststörungen (Barrett/Dadds/Rapee 1996), Entwicklungsstörungen (Schreibman/Kaneko/Koegel 1991) ebenso wie bei Verhaltensstörungen (siehe Sanders 1996; Taylor/Biglan 1998) eignet. Die vorliegenden Interventionsstudien dokumentieren mittlere bis starke Effektgrößen (Serketich/Dumas 1996).

Positive Effekte von Triple P konnten auch im Rahmen eines niedrigschwelligen Angebots mittels einer Fernsehausstrahlung nachgewiesen werden (Sanders/Mont-gomery/Brechman-Toussaint 2003), woraus gefolgert werden kann, dass bereits minimale Interventionen über Medien Änderungen in der Erziehungspraxis und folglich im Verhalten des Kindes bewirken können (vgl. auch WebsterStratton 1994).

Die Gruppenversion des Triple P-Programms wurde in einem Großversuch mit 1673 Familien in Ost-Perth, Westaustralien, evaluiert (Turner/Markie-Dadds/ Sanders 1997). Die Ergebnisse der Studie zeigen, dass Kinder, deren Eltern einen Triple P-Kurs besuchten, nach der Intervention signifikant weniger externalisierendes und oppositionelles Verhalten aufwiesen. Vor der Intervention lag das externalisierende Verhalten von $42 \%$ der Kinder im klinischen Bereich, durch die Teilnahme am Triple P für Gruppen konnte diese Zahl auf 20\% halbiert werden. Nach dem Training zeigten Eltern eine verbesserte Eltern-Kind-Interaktion und weniger Negativität in der Erziehung. Außerdem konnte die Anwendung von dysfunktionalem Erziehungsverhalten, Ehekonflikte, Erziehungsstress und Depression reduziert und die Zufriedenheit in der Partnerschaft verbessert werden. Die Robustheit dieser Befunde wurde in weiteren Replikationsstudien in Australien (Brisbane, Queensland; Sydney, New South Wales), Deutschland (Braunschweig) und der Schweiz (Fribourg) untersucht (Bertram et al. 2003; Ledermann/Cina/Meyer/Gabriel/Bo-denmann 2004; Zubrick et al. 2005). Neben den Gruppeninterventionen zeigen auch Selbsthilfeinterventionen mittels Telefonkontakten oder dem Elternarbeitsbuch vielversprechende positive Effekte (z.B. Connell/Sanders/Markie-Dadds 1997; Markie-Dadds/Sanders 2003; Markie- 
Dadds/Sanders/Smith

1997;

Sanders/

Lynch/Markie-Dadds 1994).

Neuere Studien testen vermehrt verschiedene Varianten von Triple P (StandardVariante, Erweitertes Triple P) (z.B.Sanders/Markie-Dadds/Tully/Bor 2000). Gute Erfolge von Triple P konnten auch bei spezifischen Gruppen nachgewiesen werden. So zeigt eine Untersuchung von Sanders und McFarland (2000), dass Triple P bei depressiven Eltern von Kindern mit oppositionellem Verhalten eine gute Wirksamkeit unter Beweis stellen konnte. 


\section{Kulturübertragbarkeit von Triple $\mathrm{P}$}

Insgesamt konnte die Wirksamkeit und Effektivität verschiedener Programmteile des Triple P-Systems in mehreren Ländern überzeugend nachgewiesen werden. Für eine Implementierung in einen anderen Kulturkreis (z.B. im deutschsprachigen Raum in Europa) sind jedoch eigene Studien in den betreffenden Ländern von Bedeutung, da es zu überprüfen gilt, ob Triple $\mathrm{P}$ auch in diesem Kulturkreis akzeptiert wird und wirksam ist. Kontroversen im deutschsprachigen Raum bezüglich einzelner Teile und der Arbeitsweise des Programms (Deegener/ TschöpeScheffler 2004; Tschöpe-Scheffler 2003, 2004, 2005) weisen auf die Relevanz hin, dass die Qualität von Elternprogrammen überprüft und die Akzeptanz von Elternkursen belegt werden müssen. Gemäß Perrez (1980) müssen präventive Programme drei Kriterien erfüllen: a) die Programme müssen auf die Reduktion von Verhaltensstörungen fokussieren, eine Verbesserung der elterlichen Erziehungspraktiken, Verminderung der familiären Risikofaktoren und Stärkung von sozialen Schutzfaktoren bewirken, gleichzeitig sollen stabile Effekte nachgewiesen werden und das Programm eine hohe Akzeptanz bei den Eltern erfahren, (b) das Programm soll auf Risiko- und Schutzfaktoren fokussieren und theoretisch fundiert sein sowie (c) empirisch fundiert sein (bezüglich ihrer Wirksamkeit überprüft). Während die ersten beiden von Perrez (1980) geforderten Kriterien erfüllt sind, muss die Akzeptanz sowie Wirksamkeit des im englischsprachigen Raum entwickelten Programms Triple P im deutschsprachigen Raum überprüft werden. Das Programm Triple $\mathrm{P}$, insbesondere das Elterngruppenprogramm, wird aktuell in randomisierten Kontrollgruppenstudien in Deutschland und der Schweiz evaluiert. Neben diesen Studien werden im Rahmen der Durchführung der Triple PElterngruppen Kursevaluationen durchgeführt und Eltern bezüglich der Zufriedenheit mit Triple $\mathrm{P}$ befragt. Im Folgenden werden erste Ergebnisse zu dieser Fragestellung und zu den von den Eltern selbst eingeschätzten Veränderungen präsentiert.

\section{Studie zur Zufriedenheit mit Triple P und der Akzeptanz des Programms im deutschsprachigen Raum}

1998 wurde Triple P in Deutschland, drei Jahre später in der Schweiz eingeführt. Im vorliegenden Beitrag wird (a) die Zufriedenheit der Eltern mit den Triple PKursen (Ebene 4 Gruppe) sowie (b) deren Akzeptanz, operationalisiert über die Anwendung der Triple P-Elemente nach Abschluss des Programms, dargestellt. Zurückgegriffen wird auf Angaben von Eltern aus Deutschland und der Schweiz, die an einem Triple P-Elternkurs teilgenommen haben und Rückmeldung zu den Kursen gaben. 
Methode

\section{Stichprobenrekrutierung}

In Deutschland wurden den Eltern von den Triple P-Kursleitern vor und nach dem Training Fragebögen ausgehändigt. Die Eltern konnten die Fragebögen den Kursleitern abgeben, welche diese an Triple P Deutschland (PAG) weiterleiteten. Außer zu Alter und Geschlecht des Kindes wurden keine demographischen Angaben erhoben. Die vorliegenden Daten wurden zwischen Mitte 2000 bis 2004 erhoben.

In der Schweiz erhielten alle Eltern, die von März 2002 bis April 2003 an einem Triple P-Elternkurs teilnahmen, vor der Teilnahme am Triple P-Programm von den Kursleitern einen Fragebogen zugesandt, mit der Bitte, diesen auszufüllen, an das Institut für Familienforschung und -beratung zuzusenden und damit an der Evaluation des Programms im Rahmen des Präventionsprojekts „Triple P zur Förderung der Erziehungskompetenz von Eltern “ (mit finanzieller Unterstützung des Bundesamtes für Sozialversicherung, BSV) teilzunehmen. Die Eltern, die sich bereit erklärten, wurden nach Beendigung des Trainings und, soweit die Anschrift bekannt gegeben wurde, ein halbes und ein Jahr nach Abschluss des Trainings erneut mittels Fragebogen befragt. Den Eltern wurde je ein Fragebogen für jeden Elternteil (Mutter und Vater) zugesandt und zwar unabhängig davon, ob beide Elternteile oder nur ein Elternteil an dem Training teilnahm. In dem Zeitraum fanden 72 Triple P-Kurse statt, an welchen rund 850 Familien teilgenommen haben. Daten von 405 Schweizer Eltern gehen in die folgenden Analysen ein.

\section{Stichprobe}

Bei beiden Stichproben handelt es sich um anfallende Stichproben, da die Teilnahme an der Triple P-Evaluation auf freiwilliger Basis erfolgte. Insgesamt liegen Daten von 1695 Eltern vor (1290 aus Deutschland, 405 aus der Schweiz).

An der Begleitevaluation von Triple P in der Schweiz nahmen 306 Mütter und 99 Väter teil. 89,6\% der Eltern waren verheiratet, 5\% unverheiratet und 4,5\% getrennt oder geschieden. 93,2\% lebten mit ihrer/m Partner/in zusammen. Die Partnerschaft dauerte im Schnitt 11,3 Jahre $(S D=5.0$, Range $=1-25)$. 6,9\% besuchten die reguläre Schulzeit, $48,7 \%$ verfügen über einen Berufsschulabschluss, $20 \%$ schlossen ein Gymnasium ab und 24,5\% besitzen einen Hochschul- bzw. Universitätsabschluss. 14,5\% gaben an, über ein geringes Einkommen zu verfügen (unter 60.000 Schweizer Franken), 51\% über ein mittleres Einkommen und 34,2\% r ein hohes familiäres Einkommen (über 100.000 Schweizer Franken).

\section{Erfassungsinstrumente}

Neben Fragen zum Verhalten des Kindes sowie dem Erziehungsverhalten der Eltern wurden die Eltern nach Abschluss des Programms mittels eines Fragebogen bezüglich der Zufriedenheit mit dem Programm (Kursbeurteilung, 11 Items, 7stufige Likert-Skalen), selbst wahrgenommene Verbesserungen in der Familie (5 Items, 7-stufige Likert-Skalen) und Nützlichkeit der Strategien für die familiäre 
Situation (erhoben für jede Strategie, 1 = gar nicht hilfreich bis $4=$ sehr hilfreich) befragt.

\section{Ergebnisse}

\section{Ergebnisse zur Zufriedenheit mit Triple $\mathrm{P}$}

Die Ergebnisse zur Zufriedenheit mit den Triple P-Kursen zeigen, dass das Programm bei den Eltern sehr gut ankommt, und dass diese angeben, in hohem Maße vom Programm profitiert zu haben. So sind 95,2\% der Eltern zufrieden bis sehr zufrieden mit dem Programm (Deutschland: 93,4\%; Schweiz: 91,3\%), lediglich $1,3 \%$ waren unzufrieden bis sehr unzufrieden mit dem Angebot (Deutschland: 0,7\%; Schweiz: 3,2\%). 93,6\% bewerten die Qualität des Programms, das sie erhalten haben, als gut bis hervorragend (Deutschland: 95,2\%; Schweiz: 88,5\%), 3\% als unbefriedigend bis ausreichend (Deutschland: 1,8\%; Schweiz: 6,8\%). 92\% der Eltern gaben an, die Hilfe bekommen zu haben, die sie erwartet haben (Deutschland: 98,3\%; Schweiz: 94,5\%), 2,6\% gaben an, diese eher nicht bis gar nicht erhalten zu haben (Deutschland: $1,7 \%$; Schweiz: 5,5\%). Diese insgesamt positive Einschätzung der Kursqualität spiegelt sich in den Angaben der Eltern wider, dass sie auf Triple $\mathrm{P}$ zurückkommen würden, sollten sie wieder einmal erzieherische Hilfe beanspruchen (94,1\%; Deutschland: 97,3\%; Schweiz: 90,8\%).

Der Vergleich der Evaluationen der Kurse in Deutschland und der Schweiz zeigt, dass in den vorliegenden Kursrückmeldungen die Triple P-Kurse in Deutschland leicht besser beurteilt werden als in der Schweiz, dass jedoch in beiden Ländern eine sehr hohe Zufriedenheit mit Triple P beobachtet werden kann (siehe Tabelle 4). Keine Unterschiede zwischen den Ländern liegen bezüglich der Rückmeldung der Eltern zum Fortschritt des Kindes vor. Die Betrachtung des Eta ${ }^{2}$ weist auf mittlere Effektstärken hin $\left(\mathrm{Eta}^{2}=<.06\right)$. 
Tabelle 4: $\quad$ Vergleich der Einschätzung der Eltern bezüglich der Qualität des erhaltenen Triple P-Trainings in Deutschland und der Schweiz

\begin{tabular}{|c|c|c|c|c|c|c|c|}
\hline & \multicolumn{2}{|c|}{ Deutschland } & \multicolumn{2}{|c|}{ Schweiz } & & & \\
\hline & \multicolumn{2}{|c|}{$\mathrm{N}=1290$} & \multicolumn{2}{|c|}{$\mathrm{N}=405$} & & & \\
\hline & M & SD & M & SD & \multicolumn{2}{|c|}{$\mathrm{F}$} & $\mathrm{Eta}^{2}$ \\
\hline Qualität des Triple P-Programms & 5.70 & .89 & 5.35 & 1.05 & 44.00 & *** & .03 \\
\hline Zufriedenheit mit dem Triple P-Programm & 6.03 & .93 & 5.61 & 1.06 & 59.25 & *** & .03 \\
\hline Erhalt der erwarteten Hilfe & 5.80 & .94 & 5.49 & 1.08 & 30.94 & $\star \star \star *$ & .02 \\
\hline Umfang der Hilfe & 5.91 & .95 & 5.43 & 1.03 & 75.10 & *** & .04 \\
\hline Kompetenz der Kursleitung & 6.52 & .73 & 5.97 & 1.18 & 125.91 & 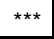 & .07 \\
\hline Unterstützung durch die Kursleitung & 6.66 & .67 & 6.34 & .98 & 53.39 & $* * *$ & .03 \\
\hline Kursatmosphäre & 6.31 & .85 & 6.03 & .98 & 29.16 & $\star \star \star *$ & .02 \\
\hline Bedürfnissen des Kindes entsprochen & 5.50 & .96 & 5.01 & .92 & 80.96 & $* \star *$ & .05 \\
\hline $\begin{array}{l}\text { Erfüllung der Bedürfnisse der Kurs- } \\
\text { teilnehmer }\end{array}$ & 5.60 & .94 & 5.12 & .92 & 82.84 & $\star \star \star \star ~$ & .05 \\
\hline Weitergabe von Strategien an Andere & 5.95 & 1.07 & 5.55 & 1.24 & 37.59 & $\star * *$ & .02 \\
\hline $\begin{array}{l}\text { Wieder auf Triple } \mathrm{P} \text { zurückkommen bei } \\
\text { Schwierigkeiten }\end{array}$ & 6.22 & 1.00 & 5.72 & 1.34 & 64.87 & *** & .04 \\
\hline
\end{tabular}

Anmerkung: Skala: 1 bis 7 (,gar nicht" bis ,ja völlig“).

\section{Unterstützung durch die Strategien}

Die Strategien, die in den Triple P-Kursen vermittelt wurden, werden von den Müttern und Vätern als hilfreich beziehungsweise sehr hilfreich für die Gestaltung des Erziehungsalltags erlebt. 97,7\% der Eltern (Deutschland: 97,7\%; Schweiz: 97,6\%) beurteilten die vermittelten Elemente der Sitzung 1 (Prinzipien der Positiven Beziehung, Ursachen von kindlichem Problemverhalten und Verhaltensbeobachtung) als überwiegend bis vollkommen hilfreich. Im ähnlichen Ausmaß wurden auch die anderen Sitzungen als hilfreich empfunden. So waren für 97,6\% der Eltern (Deutschland: 98\%; Schweiz: 96,7\%) die Strategien zur Förderung einer positiven Beziehung zum Kind, zur Förderung von angemessenem kindlichen Verhalten und Erlernen neuer Fertigkeiten (Sitzung 2), für 97,3\% (Deutschland: 97,6\%; Schweiz: 96,6\%) die Sitzung 3 (Umgang mit kindlichem Problemverhalten) und 90,8\% (Deutschland: 91,9\%; Schweiz:87,7\%) das Vorausplanen in Risikosituationen (Sitzung 4) überwiegend bis vollkommen hilfreich. Auch die anschließenden Telefonkontakte wurden von der großen Mehrheit der Eltern, die Rückmeldung zum Programm gaben, als hilfreich empfunden (insgesamt: 91,1\%; Deutschland: 93,3\%; Schweiz: 84,1\%). 
Tabelle 5: Vergleich der Einschätzung der Eltern bezüglich der Nützlichkeit der Triple P-Strategien zum Post-Messzeitpunkt in Deutschland und der Schweiz

\begin{tabular}{|c|c|c|c|c|c|c|c|}
\hline & \multicolumn{2}{|c|}{ Deutschland } & \multicolumn{2}{|c|}{ Schweiz } & & & \\
\hline & \multicolumn{2}{|c|}{$\mathrm{N}=1290$} & \multicolumn{2}{|c|}{$\mathrm{N}=405$} & \multirow{2}{*}{\multicolumn{2}{|c|}{$\mathrm{F}$}} & \multirow[b]{2}{*}{$\mathrm{Eta}^{2}$} \\
\hline & M & SD & M & SD & & & \\
\hline $\begin{array}{l}\text { 1. Sitzung: } \\
\text { Prinzipien der Positiven Erziehung }\end{array}$ & 3.47 & .57 & 3.46 & .56 & .05 & & .00 \\
\hline $\begin{array}{l}\text { 2. Sitzung: } \\
\text { Förderung der kindlichen Entwicklung }\end{array}$ & 3.53 & .55 & 3.40 & .56 & 14.91 & $* * *$ & .01 \\
\hline Wertvolle Zeit & 3.66 & .54 & 3.55 & 63 & 9.95 & ** & .01 \\
\hline Mit dem Kind sprechen & 3.66 & .55 & 3.53 & .66 & 15.87 & $\star * \star$ & .01 \\
\hline Zuneigung zeigen & 3.74 & .53 & 3.62 & .65 & 12.82 & $\star * *$ & .01 \\
\hline Loben & 3.81 & .43 & 3.66 & .60 & 27.69 & 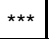 & .02 \\
\hline Aufmerksamkeit schenken & 3.69 & .52 & 3.60 & .59 & 7.64 & ** & .00 \\
\hline Ideen für spannende Beschäftigungen & 3.28 & .73 & 3.22 & .77 & 2.47 & & .00 \\
\hline Lernen am Modell & 3.32 & .74 & 3.17 & .74 & 11.42 & *** & .01 \\
\hline Beiläufiges Lernen & 3.41 & .66 & 3.32 & .70 & 5.49 & * & .00 \\
\hline Fragen-Sagen-Tun & 3.16 & .83 & 3.10 & .79 & 1.46 & & .00 \\
\hline Punktekarte & 3.29 & .94 & 3.10 & .95 & 10.97 & $* * \star$ & .01 \\
\hline $\begin{array}{l}\text { 3. Sitzung: } \\
\text { Umgang mit Problemverhalten }\end{array}$ & 3.59 & .57 & 3.39 & .59 & 18.64 & $\star \star \star *$ & .02 \\
\hline Familienregeln & 3.53 & .63 & 3.39 & .72 & 13.19 & $\star * *$ & .01 \\
\hline Direktes Ansprechen & 3.69 & .51 & 3.58 & .60 & 10.14 & $\star \star \star *$ & .01 \\
\hline Absichtliches Ignorieren & 3.28 & .77 & 3.14 & .78 & 9.46 & ** & .01 \\
\hline Klare ruhige Anweisungen & 3.73 & .46 & 3.62 & .58 & 14.11 & $* \star *$ & .01 \\
\hline Logische Konsequenzen & 3.69 & .51 & 3.59 & .63 & 10.77 & $* * *$ & .01 \\
\hline Stiller Stuhl & 2.99 & 1.06 & 2.75 & 1.03 & 14.03 & $\star * *$ & .01 \\
\hline Auszeit & 3.23 & .93 & 3.07 & .94 & 7.86 & ** & .01 \\
\hline $\begin{array}{l}\text { 4. Sitzung: } \\
\text { Vorausplanen }\end{array}$ & 3.33 & .65 & 3.24 & .70 & 4.77 & * & .00 \\
\hline $\begin{array}{l}\text { 5. bis } 8 \text {. Sitzung: } \\
\text { Telefonkontakte }\end{array}$ & 3.42 & .69 & 3.13 & .77 & 43.15 & $\star * \star$ & .03 \\
\hline
\end{tabular}

Anmerkung: Skala: 1 = gar nicht hilfreich bis $4=$ vollkommen hilfreich.

${ }^{*} \mathrm{p}<.05 ;{ }^{* \star} \mathrm{p}<.01 ;{ }^{* \star *} \mathrm{p}<.001$.

Werden die Angaben der Eltern aus der Schweizer Stichprobe über ein Jahr verfolgt, zeigt sich, dass auch ein Jahr nach Abschluss des Trainings die Eltern die Strategien immer noch hilfreich finden (siehe Tabelle 6). 
Tabelle 6: $\quad$ Einschätzung der Trainingsinhalte sechs Monate und 1 Jahr nach Teilnahme am Triple P-Kurs (in Prozent)

\begin{tabular}{|c|c|c|c|c|c|c|}
\hline & \multicolumn{3}{|c|}{6 Monate } & \multicolumn{3}{|c|}{1 Jahr } \\
\hline & $\begin{array}{c}\text { Gar } \\
\text { nicht/eher } \\
\text { nicht }\end{array}$ & $\begin{array}{l}\text { Über- } \\
\text { wiegend }\end{array}$ & $\begin{array}{l}\text { Voll- } \\
\text { kommen }\end{array}$ & $\begin{array}{c}\text { Gar } \\
\text { nicht/eher } \\
\text { nicht }\end{array}$ & $\begin{array}{c}\text { Über- } \\
\text { wiegend }\end{array}$ & $\begin{array}{c}\text { Voll- } \\
\text { kommen }\end{array}$ \\
\hline $\begin{array}{l}\text { 1. Sitzung: } \\
\text { Prinzipien der Positiven Erziehung }\end{array}$ & 4.81 & 62.20 & 32.99 & 3.73 & 57.09 & 39.18 \\
\hline $\begin{array}{l}\text { 2. Sitzung: } \\
\text { Förderung der kindlichen Entwick- } \\
\text { lung }\end{array}$ & 7.33 & 60.34 & 32.33 & 4.07 & 63.80 & 32.13 \\
\hline Wertvolle Zeit & 3.57 & 47.08 & 49.35 & 2.41 & 45.17 & 52.41 \\
\hline Mit dem Kind sprechen & 5.52 & 34.09 & 60.39 & 4.50 & 40.83 & 54.67 \\
\hline Zuneigung zeigen & 4.21 & 30.10 & 65.70 & 2.42 & 32.87 & 64.71 \\
\hline Loben & 4.87 & 35.06 & 60.06 & 2.41 & 34.48 & 63.10 \\
\hline Aufmerksamkeit schenken & 5.59 & 39.47 & 54.93 & 3.13 & 41.32 & 55.56 \\
\hline $\begin{array}{l}\text { Ideen für spannende Beschäfti- } \\
\text { gungen }\end{array}$ & 21.24 & 54.90 & 23.86 & 19.51 & 52.61 & 27.87 \\
\hline Lernen am Modell & 23.26 & 51.50 & 25.25 & 25.37 & 50.74 & 23.90 \\
\hline Beiläufiges Lernen & 9.45 & 55.70 & 34.85 & 12.10 & 55.87 & 32.03 \\
\hline Fragen-Sagen-Tun & 27.42 & 51.84 & 20.74 & 29.64 & 49.29 & 21.07 \\
\hline Punktekarte & 37.37 & 34.34 & 28.28 & 34.06 & 35.14 & 30.80 \\
\hline $\begin{array}{l}\text { 3. Sitzung: } \\
\text { Umgang mit Problemverhalten }\end{array}$ & 5.81 & 63.23 & 30.97 & 2.65 & 58.28 & 39.07 \\
\hline Familienregeln & 14.01 & 50.81 & 35.18 & 13.03 & 45.77 & 41.20 \\
\hline Direktes Ansprechen & 4.56 & 49.51 & 45.93 & 6.32 & 42.81 & 50.88 \\
\hline Absichtliches Ignorieren & 29.74 & 46.73 & 23.53 & 28.32 & 46.15 & 25.52 \\
\hline Klare ruhige Anweisungen & 3.87 & 41.61 & 54.52 & 5.96 & 41.75 & 52.28 \\
\hline Logische Konsequenzen & 7.89 & 42.43 & 49.67 & 7.39 & 40.49 & 52.11 \\
\hline Stiller Stuhl & 43.69 & 35.84 & 20.48 & 47.43 & 29.04 & 23.53 \\
\hline Auszeit & 26.26 & 41.08 & 32.66 & 26.79 & 39.29 & 33.93 \\
\hline $\begin{array}{l}\text { 4. Sitzung: } \\
\text { Vorausplanen }\end{array}$ & 19.01 & 50.70 & 30.28 & 19.14 & 45.70 & 35.16 \\
\hline $\begin{array}{l}\text { 5.bis 8. Sitzung: } \\
\text { Telefonkontakte } \\
\end{array}$ & 33.20 & 44.67 & 22.13 & 31.96 & 46.12 & 21.92 \\
\hline
\end{tabular}

\section{Ergebnisse zur Akzeptanz von Triple P}

Es wird davon ausgegangen, dass Triple $\mathrm{P}$ dann als akzeptiert betrachtet werden kann, wenn die Eltern die im Programm gelernten Methoden auch im Alltag wirklich anwenden. Die Ergebnisse hierzu zeigen ein ebenfalls sehr erfreuliches Bild.

Von den 17 vermittelten Strategien werden acht auch ein Jahr nach dem Kurs (Follow-up 2) noch von über $90 \%$ der Teilnehmer/-innen als nützlich beurteilt. Dazu gehören vor allem auch die präventiven Strategien zur Vermeidung von Problemverhalten wie z.B. „Wertvolle Zeit“, „mit dem Kind reden“, „Loben“, 
„Aufmerksamkeit schenken“ und „Zuneigung zeigen“, die speziell der Beziehung zwischen Eltern-Kind und der kindlichen Entwicklung dienen. Auch die Strategien zum angemessenen Umgang mit Problemverhalten werden angewendet. Vor allem die Strategien „Direktes Ansprechen“, „Klare, ruhige Anweisungen“ und „Logische Konsequenzen" werden als besonders hilfreich empfunden. Als am wenigsten hilfreich werden die Strategien "Stiller Stuhl" und „Punktekarte“ beurteilt, beides Strategien, die sehr gezielt eingesetzt werden müssen.

Ergebnisse zu selbst eingeschätzten positiven Veränderungen in der Familie infolge von Triple $\mathrm{P}$

Neben der generellen Zufriedenheit der Eltern mit dem Programm finden sich auch konkrete positive Veränderungen im Erziehungsverhalten im Alltag. Gemäß ihrer eigenen Einschätzung geben die meisten Eltern nach dem Kurs an, kompetenter bei der Erziehung der Kinder zu sein, und dass das Verhalten des Kindes besser ist. 97,4\% der Eltern (Deutschland: 98,3\%; Schweiz: 94,5\%) berichten, dass das Programm ihnen geholfen hat, mit dem Verhalten ihres Kindes besser umzugehen. 91,9\% der Eltern (Deutschland: 93,8\%; Schweiz: 86\%) stellen nach dem Kurs moderate bis starke Verbesserungen bezüglich des Verhaltens ihres Kindes fest.

Die positiven Effekte von Triple P wirken sich auch auf das Familienleben generell aus. 89,4\% der Eltern (Deutschland: 92,4\%; Schweiz: 80\%) sind der Ansicht, dass der Kurs ihnen geholfen hat, Probleme in der Familie generell besser bewältigen zu können. Erfreulich ist, dass in vielen Fällen (66,4\%; Deutschland: 68,6\%; Schweiz: 62,6\%) sogar die Partnerschaft von den neuen Kompetenzen/Ressourcen der Eltern im Umgang mit den Kindern zu profitieren vermag (siehe Tabelle 7).

Zusammenfassend kann gefolgert werden, dass die Eltern, die Rückmeldung zum Triple P-Training gaben, insgesamt sehr zufrieden mit der Qualität und dem Inhalt sind, die im Kurs gelernten Strategien als hilfreich in ihrem Alltag betrachten und von positiven Veränderungen im Erziehungsverhalten und dem Verhalten des Kindes berichten. Es liegen jedoch nicht von allen Eltern, die einen Triple PKurs besucht haben, Kursevaluationen vor. Es ist daher von Bedeutung, die hier dargestellten Ergebnisse in anderen Studien zu replizieren. Erste Hinweise auf die Gütigkeit der Ergebnisse zeigen Kontrollgruppenstudien aus Deutschland und der Schweiz (Cina et al. 2004; Heinrichs et al. 2006 a). 
Tabelle 7: Vergleich der selbst eingeschätzten Veränderungen zum PostMesszeitpunkt in Deutschland und der Schweiz

\begin{tabular}{|l|r|r|r|r|r|r|r||}
\hline & \multicolumn{2}{|c|}{ Deutschland } & \multicolumn{2}{|c|}{ Schweiz } & & & \\
\hline & \multicolumn{2}{|c|}{$\mathrm{N}=1290$} & \multicolumn{2}{|c|}{$\mathrm{N}=405$} & & & \\
\hline & \multicolumn{1}{|c|}{$\mathrm{M}$} & \multicolumn{1}{c|}{$\mathrm{SD}$} & $\mathrm{M}$ & \multicolumn{1}{c|}{$\mathrm{SD}$} & \multicolumn{2}{|c||}{$\mathrm{F}$} & $\mathrm{Eta}^{2}$ \\
\hline Verhalten des Kindes & 5.78 & .85 & 5.46 & .89 & 42.69 & $* * *$ & .02 \\
\hline Fortschritt des Kindes & 5.74 & .96 & 5.67 & .99 & 1.52 & & .00 \\
\hline $\begin{array}{l}\text { Verbesserung im Umgang mit dem Ver- } \\
\text { halten des Kindes }\end{array}$ & 6.45 & .84 & 6.00 & 1.07 & 75.64 & $* * *$ & .04 \\
\hline Hilfe bei Problemen innerhalb der Familie & 5.98 & 1.10 & 5.38 & 1.28 & 83.15 & $* * *$ & .05 \\
\hline Nutzen für die Partnerschaft & 4.76 & 1.29 & 4.59 & 1.34 & 4.38 & $*$ & .00 \\
\hline
\end{tabular}

Anmerkung: Skala 1 bis 7 (,nein, gar nicht“ bis ,ja, völlig“)

${ }^{\star} \mathrm{p}<.05 ;{ }^{* * *} \mathrm{p}<.001$.

\section{Wirksamkeit von Triple P im deutschsprachigen Raum}

Neben der Frage, wie zufrieden die Eltern mit Triple P sind und wie gut sie deren Methoden im Alltag akzeptieren und anwenden, interessiert uns vor allem auch die Frage, ob Triple P auch wirksam ist und zu einer besseren Erziehungskompetenz der Eltern und einem besseren Befinden der Kinder und Eltern führt. Zur Klärung dieser Fragen laufen zurzeit in Deutschland und der Schweiz mehrere randomisierte Kontrollgruppenstudien.

\section{Deutschland}

In der prospektiven randomisierten Kontrollgruppenstudie „Zukunft Familie“ I (Fünf-Jahres-Längsschnittstudie) wird die Wirksamkeit universeller Präventionsmaßnahmen (angewendet wurde das Positive Elternprogramm Triple P als Gruppenvariante, TPG) für Familien mit Kindern im Alter von 2,5 bis 6 Jahren evaluiert (Heinrichs et al. 2006 a). Über städtische Kindertagesstätten konnten 280 Familien rekrutiert werden. 186 Familien wurde das Angebot gemacht, an einem Triple P-Training teilzunehmen, davon nahmen 144 Familien das Angebot an. 94 Familien wurden der Kontrollgruppe zugeteilt.

Die Ergebnisse zeigen, dass die Rückmeldungen der Eltern sich mit den oben genannten Ergebnissen decken. 90\% der Eltern schätzten die Qualität des Trainings als gut bis hervorragend ein, $81 \%$ der Eltern geben an, dass sich seit dem Besuch des Programms die Beziehung zum Kind verbessert habe, $79 \%$ sind der Ansicht, das kindliche Verhalten habe sich gebessert und weitere $85 \%$ geben an, mit Pro-blemen in der Familie besser umgehen zu können. $43 \%$ meinen, dass auch die Partnerschaft vom Triple P-Programm profitiert habe.

Bezüglich des Erziehungsverhaltens der Eltern (erhoben anhand des EFB, Miller 2001) zeigte sich, dass diese ein Jahr nach ihrer Teilnahme am Triple PGrup-penprogramm weniger häufig ungünstiges Erziehungsverhalten zeigen und insgesamt ein positiveres Erziehungsverhalten aufweisen. Hinsichtlich des kindli- 
chen Problemverhaltens (erhoben anhand des CBCL, Arbeitsgruppe Deutsche Child Behavior Checklist 2000) gelang es in der Interventionsgruppe (insgesamt 219 Zweielternfamilien, Triple P: 129 Familien), den Anteil an Kindern mit klinisch und grenzwertig auffälligem externalem Problemverhalten von 17,6\% (vor dem Training) auf 4,8\% (1 Jahr nach dem Training) zu reduzieren, während sich in der Kontrollgruppe kaum Veränderungen ergaben (Prävalenzrate vor dem Training: 13,6\%, 1 Jahr später: 12,5\%). Neben dem externalisierenden konnte auch eine Reduktion bei der Prävalenz von internalisierendem Problemverhalten bei der Interventionsgruppe Triple $\mathrm{P}$ von 20,6\% (vor der Teilnahme am Training) auf 11,1\% (1 Jahr nach dem Training ) erreicht werden (Kontrollgruppe: 11,4\% auf 9,1\%). Neben einer Reduktion der Prävalenzrate zeigte die Interventionsgruppe eine geringere Inzidenzrate (externalisierendes Problemverhalten: 1,9\% vs. 3,4\%; interalisierendes Problemverhalten: 0,8\% vs. 4,6\%). Die Effektivitäts- und Disseminationsstudie „Zukunft Familie“ II zeigt analoge Ergebnisse (Heinrichs et al. 2006 b). Zusammenfassend lässt sich festhalten, dass das Elterntraining längerfristig eine bedeutsame Reduktion ungünstigen Erziehungsverhaltens bewirkt und damit die theoretische Grundvoraussetzung für eine längerfristige Reduktion kindlicher Verhaltensprobleme erfüllt.

\section{Schweiz}

In der Schweiz werden in einer randomisierten Kontrollgruppenstudie die Effekte des Triple P-Gruppenprogramms (TPG) und dem Freiburger Stresspräventionstraining für Paare (FSPT, siehe Bodenmann 2000) über ein Jahr untersucht. Beide Interventionen sind präventive Programme, die auf bekannte familiäre Risikofaktoren für kindliches Problemverhalten fokussieren, d.h. Defizite in der Erziehungskompetenz (Triple P) oder Partnerschaftskonflikte (FSPT). Die Studie hat zum Ziel, Aufschluss darüber zu geben, welche differentiellen Effekte mittels den beiden Präventionsangeboten zur Reduktion von Stress bei Familien und damit dem Befinden und Verhalten des Kindes erzielt werden können. Je 50 Familien mit Kindern im Alter zwischen 2 und 12 Jahren wurden zufällig auf eine Kontrollgruppe oder auf eine der beiden Interventionsmethoden Triple PGruppenprogramm oder FSPT verteilt.

Ergebnisse zeigen auch hier, dass das Triple P-Programm bei den Eltern sehr gut ankommt, und dass diese angeben, in hohem Maße vom Programm profitiert zu haben. 95\% der Eltern geben an, dass sie mit dem Programm sehr zufrieden waren und rund $94 \%$ der Teilnehmer/-innen würden Triple P weiterempfehlen, wovon dies $63 \%$,,auf jeden Fall“ täten, was als Indikator für eine hohe Kurszufriedenheit und eine gute Akzeptanz von Triple P bei den teilnehmenden Eltern gewertet werden kann. Erfreulich ist auch, dass rund ein Drittel der Eltern angibt, dass sie die gelernten Inhalte im Alltag auch regelmäßig und konsequent anwenden. Weitere 60\% geben an, dass sie von den Triple P-Methoden im Alltag ab und zu Gebrauch machen, was die Nützlichkeit und die Akzeptanz der gelernten Strategien verdeutlicht. Lediglich 10\% wenden die gelernten Strategien nach eigenen Angaben selten bis nie an. Subjektiv berichten die Eltern von einer Verbesserung 
bezüglich des Verhaltens des Kindes, dem eigenen Erziehungsverhalten, einer günstigeren Paarkommunikation als auch von vermindertem Stress in der Familie.

Diese positiven Effekte konnten mehrheitlich bei beiden Präventionsprogrammen nachgewiesen werden, während die Kontrollgruppe keine signifikanten Veränderungen aufwies (Ledermann/Cina/Meyer/Gabriel/Bodenmann 2004).

Die ersten Resultate ein Jahr nach der Teilnahme am Training machen deutlich, dass die Teilnahme am Triple P zu einer deutlichen Verbesserung im Erziehungsverhalten führt (erhoben anhand des EFB, Miller 2001). Die Eltern, die an einem Triple P-Kurs teilnahmen, zeigten nach Teilnahme am Training verglichen mit der Kontrollgruppe signifikant günstigeres Erziehungsverhalten als vor Kursbeginn. Sie reagierten weniger nachsichtig und geben an, weniger häufig in Erziehungssituationen überzureagieren (Ledermann et al. 2004). Auch konnte die Prävalenzrate von kindlichem Problemverhalten (erhoben anhand des ECBI, Eyberg 1992) halbiert werden. Von ursprünglich 39\% der Kinder lagen ein Jahr nach Teilnahme am Training noch $17 \%$ über dem Cut-off-Bereich.

\section{Diskussion}

Triple $\mathrm{P}$ versucht ein Gleichgewicht zwischen einem kind- und elternzentrierten Ansatz zu finden, indem es den Eltern dabei hilft, die Fertigkeiten und Kompetenzen, die ihre Kinder erwerben sollen, zu erkennen, und diese auf eine fördernde Art und Weise zu entwickeln helfen (z.B. Sprachfertigkeiten, emotionale Selbstregulation, Unabhängigkeit, Selbständigkeit und Problemlösefertigkeiten). Triple P stellt ein modernes Programm dar, welches auf dem aktuellen Wissensstand bezüglich Risiko- und Protektivfaktoren sowie wirksamen Interventionsmöglichkeiten basiert. Die Förderung der Autonomie und Selbständigkeit der Eltern durch Stärkung ihrer Erziehungsfertigkeiten sowie die Berücksichtigung der Belange des Kindes stehen im Mittelpunkt des Programms.

Das Programm hat sich in vielen Studien vor allem aus dem englischsprachigen Raum als wirksames Präventions- und Interventionsangebot erwiesen. Durch das Training der Erziehungsstrategien, die im Programm Triple P benutzt werden, konnten die Verhaltensprobleme des Kindes, welche meist schon über einige Zeit andauerten, verringert werden. Parallel zu den primären Behandlungseffekten beim Kind replizierten verschiedene Studien auch die Verbesserungen hinsichtlich der individuellen Schwierigkeiten der Eltern (insbesondere der Mutter). Die gefundenen Ergebnisse sind für alle Triple P-Programme - selbstangeleitete und telefongestützte, Standard- und Gruppenprogramm, erweiterte Triple PInterventionen - statistisch zuverlässig und klinisch bedeutsam. Sie bestätigen die Annahme, dass Erziehungsprogramme mit unterschiedlichen Intensitätsstufen erfolgreich sein können.

In diesem Zusammenhang sind Studien von Bedeutung, die die Wirksamkeit des Programms im jeweiligen Sprachraum überprüfen und die Ergebnisse der Studien, die im vor allem englischsprachigen Raum durch die Autorengruppe von Triple $\mathrm{P}$ durchgeführt wurden, replizieren. Erste Ergebnisse liegen aus dem 
deutschsprachigen Raum für das Triple P-Gruppenprogramm (TPG) vor. So zeigen die Kursevaluationen in Deutschland und der Schweiz, basierend auf freiwilligen Rückmeldungen der Eltern, dass die meisten Eltern sehr zufrieden sind mit dem Kursangebot, die in Triple P gelernten Methoden im Alltag anwenden und subjektive Verbesserungen im Verhalten des Kindes und ihrem eigenen Verhalten wahrnehmen.

Da nicht ausgeschlossen werden kann, dass nur diejenigen Eltern Rückmeldung gaben, die mit dem Programm zufrieden waren (was allerdings als unwahrscheinlich gelten darf, da Personen erfahrungsgemäss sehr gerne zu Kursen Rückmeldung geben, die ihren Erwartungen nicht entsprochen haben, im Besonderen wenn sie dafür bezahlt haben), sind Ergebnisse der elterlichen Zufriedenheit aus kontrollierten Vergleichsstudien von Bedeutung. Kontrollierte Kontrollgruppenstudien aus Deutschland (Heinrichs et al. 2006 a und b) und der Schweiz (Cina/Ledermann/

Meyer/Gabriel/Bodenmann 2004; Ledermann et al. 2004) zeigen, dass auch Eltern, die einem Triple P-Kurs zugeteilt werden, von einer ähnlichen Zufriedenheit berichten. Diese Studien zeigen, dass Triple P auch im deutschsprachigen Raum eine gute Effektivität aufweist, Eltern nach Teilnahme an dem Triple PGruppenprogramm ein positiveres Erziehungsverhalten anwenden und die Prävalenz und Inzidenzrate von kindlichem Problemverhalten durch Triple P gesenkt werden kann.

Diese sehr erfreulichen Ergebnisse widerlegen eindrücklich die im deutschen Sprachraum immer wieder an Triple $\mathrm{P}$ geäußerten Kritiken. Triple $\mathrm{P}$ wird von den Eltern nicht als Dressur ihrer Kinder erfahren, sondern als eigene Ressourcenstärkung und Verminderung von Stress; dies ermöglicht, angemessen auf die Kinder eingehen zu können.

Inzwischen hat sich Triple P in verschiedenen Ländern zu einem umfassenden mehrstufigen Unterstützungssystem in Erziehungs- und Familienfragen entwickelt und es ist zu hoffen, dass auch im deutschsprachigen Raum Triple P allen Eltern zur Verfügung gestellt werden kann, die von diesem Angebot profitieren möchten. Modifizierte Programme in anderen Sprachen oder kulturell angepasste Programme für Minderheiten (z.B. Migranten, Familien in Armut etc.) sind gefordert. Fundamentales Ziel von Triple P bleibt die Entwicklung eines umfassenden, qualitativ hochstehenden, empirisch unterstützten, mehrstufigen, präventionsorientierten, uni-versellen und frei zugänglichen Erziehungsunterstützungsmodells. Um dieses Ideal zu erreichen sind Forschungsarbeiten erforderlich, die aufzeigen, wer wie auf welche Darbietungsart reagiert und wie Familien angesprochen werden können, die üblicherweise von Präventionsprogrammen schlecht erreicht werden. Triple P bietet über das Bestehende hinaus vielversprechende Perspektiven, die für Praktiker ebenso wie für Eltern von hohem Interesse sind. 


\section{Literatur}

Arbeitsgruppe Deutsche Child Behavior Checklist (2000). Elternfragebogen für Klein- und Vorschulkinder (CBCL 1 1 1/2 - 5). Köln: Arbeitsgruppe Kinder-, Jugend- und Familiendiagnostik (KJFD).

Bandura, A. (1977). Self-efficay: Toward a unifying theory of behavioral change. Psychological Review, 84, pp. $191-215$.

Bandura, A. (1995). Self-efficacy in changing societies. New York: Cambridge University Press.

Barkley, R. A./Guevremont, D. C./Anastopoulos, A. D./Fletcher, K. E. (1992). A comparison of three family therapy programs for treating family conflicts in adolescents with attention-deficit hyperactivity disorder. Journal of Consulting and Clinical Psychology, 60 , pp. 450-462.

Barrett, P. M./Dadds, M. R./Rapee, R. M. (1996). Family treatment of childhood anxiety: A controlled trial. Journal of Consulting and Clinical Psychology, 65, pp. 627-635.

Bertram, H./Heinrichs, N./Kuschel, A./Kessemeier, Y./Saßmann, H./Hahlweg, K. (2003). Projekt „Zukunft Familie“ Erste Ergebnisse der Rekrutierung. Verhaltenstherapie und Verhaltensmedizin, 24, pp. 187-204.

Bodenmann, G. (2000). Kompetenzen in der Partnerschaft. Freiburger Stresspräventionsprogramm für Paare. Weinheim: Juventa Verlag.

Chamberlain, P./Patterson, G. R. (1995). Discipline and child compliance in parenting. In: M. H. Bornstein. (Eds.). Handbook of parenting Vol. 4; Applied and practical parenting (pp. 205-225). Mahwah, NJ, USA: Lawrence Erlbaum Associates, Inc.

Cina, A./Ledermann, T./Meyer, J./Gabriel B./Bodenmann, G. (2004). Triple $P$ in der Schweiz: Zufriedenheit, Akzeptanz und Wirksamkeit. (Forschungsbericht Nr. 162). Fribourg: Universität Fribourg.

Coie, J. D. (1996). Prevention of violence and antisocial behavior. In: R. D. Peters/R. J. McMahon (Eds.). Preventing childhood disorders, substance abuse and delinquency (pp. 1-18). Thousand Oaks, CA, USA: Sage.

Connell, S./Sanders, M. R./Markie-Dadds, C. (1997). Self-directed behavioural family intervention for parents of oppositional children in rural and remote areas. Behaviour Modification, 21, pp. 379-408.

Crick, N. R./Dodge, K. A. (1994). A review and reformulation of social informationprocessing mechanisms in children's social adjustment. Psychological Bulletin, 115, pp. 74-101.

Cummings, E. M./Davies, P. (1994). Children and marital conflict: The impact of family dispute and resolution. New York: Guildford Press.

Dadds, M. R./Schwartz, S./Sanders, M. R. (1987). Marital discord and treatment outcome in the treatment of childhood conduct disorders. Journal of Consulting and Clinical Psychology, 55, pp. 396-403.

Deegener, G./Tschöpe-Scheffler, S. (2004). „Es wird sich in den Schlaf weinen.“ Theorie und Praxis der Sozialpädagogik, 8, pp. 18-22.

Dodge, K. A. (1986). A social information processing model of social competence in children. In: M. Perlmutter (Ed.). The Minnesota symposium on child psychology (Vol. 18). Hillsdale, NJ, USA: Erlbaum.

Dryfoos, J. G. (1990). Adolescents at risk: Prevalence and prevention. New York: Oxford University Press.

Emery, R. E. (1982). Interparental conflict and the children of discord and divorce. Psychological Bulletin, 92, pp. 310-330.

Erel, O./Burman, B. (1995). Interrelatedness of marital relations and parent-child relations: A meta-analytic review. Psychological Bulletin, 118, pp. 108-132. 
Eyberg, S. M. (1992). Assessing therapy outcome with preschool children: Progress and problems. Journal of Clinical Child Psychology, 21, pp. 306-311.

Forehand. R. L./Long. N. (1988). Outpatient treatment of the acting out child: Procedures, long term follow-up data, and clinical problems. Advances in Behaviour Research and Therapy, 10 , pp. 129-177.

Grych, J. H./Fincham, F. D. (1990). Marital conflict and children's adjustment: A cognitive contextual framework. Psychological Bulletin, 108, pp. 267-290.

Hart, B./Risley. T. R. (1995). Meaningful differences in the everyday experience of young American children. Baltimore: Paul H. Brookes Publishing Co.

Heinrichs, N./Hahlweg, K./Bertram, H./Kuschel, A./Naumann, S./Harstick, S. (2006 a). Die langfristige Wirksamkeit eines Elterntrainings zur universellen Prävention kindlicher Verhaltensstörungen: Ergebnisse aus der Sicht der Mütter und Väter. Zeitschrift für Klinische Psychologie und Psychotherapie, 35. Jhg., 4, S. 97-108.

Heinrichs, N./Hahlweg, K./Kuschel, A./Bertram, H./Harstick, S./Naumann, S. (2006 b). Triple $\mathrm{P}$ aus der Sicht der Eltern: Teilnahmeraten und Kurszufriedenheit in Abhängigkeit von soziodemographischen Charakteristika und Migration. Kindheit und Entwicklung, 15, S. 19-26.

Jacobson, N. S./Margolin, G. (1979). Marital therapy: Strategies based on social learning and behavior exchange principles. New York: Brunner/Mazel.

Ledermann, T./Cina, A./Meyer, J./Gabriel, B./Bodenmann, G. (2004). Die Wirksamkeit zweier Präventionsprogramme zur Verbesserung elterlicher Kompetenzen und kindlichen Befindens. (Forschungsbericht Nr. 163). Fribourg: Universität Fribourg.

Loeber, R./Farrington. D. P. (1998). Never too early, never too late: Risk factors and successful interventions for serious and violent juvenile offenders. Studies on Crime and Crime Prevention, 7, pp. 7-30.

Markie-Dadds, C./Sanders, M. R. (2003). Effectiveness of a self-directed program for parents of children at high and low risk of developing conduct disorder. Unpublished manuscript. University of Queensland, St Lucia.

Markie-Dadds, C./Sanders, M. R./Smith, J. I. (1997). Self-directed behavioural Family intervention for parents of oppositional children in rural and remote areas. Paper presented at the 20th National Conference of the Australian Association for Cognitive and Behaviour Therapy, Brisbane, Queensland.

Miller, Y. (2001). Erziehung von Kindern im Kindergartenalter: Erziehungsverhalten und Kompetenzüberzeugungen von Eltern und der Zusammenhang zu kindlichen Verhaltensstörungen. Dissertation an der Technischen Universität Braunschweig.

Patterson, G. R. (1982). Coersive family process. Eugene, OR: Castalia Press.

Perrez, M. (1980). Implementierung neuen Erziehungsverhaltens: Interventionsforschung im Erziehungsstil-Bereich. In: K. A. Schneewind/P. Herrmann (Hrsg.). Erziehungsstilforschung (S. 245-280). Bern: Huber.

Risley, T. R./Clark, H. B./Cataldo, M. F. (1976). Behavioral technology for the normal middle class family. In: E. J. Mash/L. A. Hamerlynch/L. C. Handy (Eds.). Behavior modification and families (pp. 34-60): New York: Brunner/Mazel.

Robins, L. N./Price, R. K. (1991). Adult disorders predicted by childhood conduct problems: Results from the NIMH Epidemiological Catchment Area Project. Psychiatry, 54, pp. 116-132.

Rutter, M. (1985). Family and school influences on behavioral development. Journal of Child Psychology and Psychiatry, 26, pp. 349-368.

Sanders, M. R. (1992). Enhancing the impact of behavioural family intervention with children: Emerging perspectives. Behaviour Change, 9, pp. 115-119.

Sanders, M. R. (1996). New directions in behavioural family intervention with children. In: T. H. Ollendick/R. J. Prinz, (Eds.). Advances in clinical child psychology, Vol. 18 (pp. 283-330). New York: Plenum Press. 
Sanders, M. R. (1998). The empirical status of psychological interventions with families of children and adolescents. In: L. L'Abate (Ed.). Family psychopathology: The relational roots of dysfunctional behavior (pp. 427-465). New York, USA: Guilford Press.

Sanders, M. R./Christensen, A. P. (1985). A comparison of the effects of child management and planned activities training across five parenting environments. Journal of Abnormal Child Psychology, 13, pp. 101-117.

Sanders, M. R./Dadds, M. R. (1982). The effects of planned activities and child management training: An analysis of selling generality. Behaviour Therapy, 13, pp. 1-11.

Sanders, M. R./Dadds, M. R. (1993). Behavioural family intervention. Boston: Allyn and Bacon, Inc.

Sanders, M. R./Duncan, S. B. (1995). Empowering families: Policy, training, and research issues in promoting family mental health in Australia. Behaviour Change, 12, pp. 109121.

Sanders, M. R./Glynn, E. L. (1981). Training parents in behavioural self-management: An analysis of generalization and maintenance effects. Journal of Applied Behaviour Analysis, 14, pp. 223-237.

Sanders, M. R./Lynch, M./Markie-Dadds, C. (1994). Every parent's workbook: A positive guide to positive parenting. Brisbane: Australian Academic Press.

Sanders, M. R./Markie-Dadds, C./Tully, L./Bor, B. (2000). The Triple P-Positive Parenting Program: A comparison of enhanced, standard and self-directed behavioural family intervention for parents of children with early onset conduct problems, Journal of Consulting and Clinical Psychology, 68, pp. 624-640.

Sanders, M. R./McFarland, M. L. (2000). The treatment of depressed mothers with disruptive children: A controlled evaluation of cognitive behavioural family intervention. $B e$ haviour Therapy, 31, pp. 89-112.

Sanders, M. R./Montgomery, D. T./Brechman-Toussaint, M. L. (2003). The mass media and child behaviour problems: The effect of a television series on child and parent outcomes. Unpublished manuscript, University of Queensland, St Lucia.

Sanders, M. R./Shepherd, R. W./Cleghorn, G./Woolford, H. (1994). The treatment of recurrent abdominal pain in children. A controlled comparison of cognitive-behavioural family intervention and standard pediatric care. Journal of Consulting and Clinical Psychology, 62, pp. 306-314.

Schreibman, L./Kaneko, W. M./Koegel, R. L. (1991). Positive affect of parents of autistic children: A comparison across two teaching techniques. Behaviour Therapy, 22, pp. 479490.

Serketich, W. J./Dumas, J. E. (1996). The effectiveness of behavioural parent training to modify antisocial behaviour in children: A meta-analysis. Behaviour Therapy, 27, pp. 171-186.

Taylor, T. K./Biglan, A. (1998). Behavioural family interventions for improving childrearing: A review of the literature for clinicians and policy makers. Clinical Child and Family Psychology, 1, pp. 41-60.

Tschöpe-Scheffler, S. (2003). Elternkurse auf dem Prüfstand. Wie Erziehung wieder Freude macht. Opladen: Leske + Budrich.

Tschöpe-Scheffler, S. (2004). Elternkurse im Vergleich. Menschenbilder, Inhalte, Methoden. Theorie und Praxis der Sozialpädagogik, 8, S. 8-13.

Tschöpe-Scheffler, S. (Hrsg.) (2005). Konzepte der Elternbildung - Ein kritischer Überblick. Opladen: Barbara Budrich.

Turner, K. M. T./Markie-Dadds, C./Sanders, M. R. (1997). Facilitator's manual for group Triple P. Brisbane, Australia; Families International Publishing.

Turner, K. M. T./Sanders, M. R./Wall, C. R. (1994). Behavioural parent training versus dietary education in the treatment of children with persistent feeding difficulties. Behaviour Change, 11, pp. 242-258. 